\title{
The Prevalence of Infection in RA Patients using Biological DMARDs in King Abdul-Aziz University Hospital Jeddah, Saudi Arabia: A cross sectional study Badreyah Ahmad Aldauig ${ }^{1}$, Khalid A. Alshehri ${ }^{1}$, Ahmed A. Alharbi ${ }^{1}$, Rana M. Bajaba ${ }^{1}$, Sarah \\ A. Alghamdi ${ }^{1}$, Suzan Attar ${ }^{2}$ \\ ${ }^{1}$ College of Medicine, King Abdulaziz University, Jeddah, 2 Department of Internal Medicine (Rheumatology), King Abdulaziz University, Jeddah, Saudi arabia \\ Correspondence: Badreyah Ahmad Aldauig, College of Medicine, King Abdulaziz University, Jeddah, Saudi Arabia, PO Box-21411 Jeddah-456 district, Saudi Arabia. \\ E-mail: Bdaryahmad12@gmail.com
}

\begin{abstract}
Background: Rheumatoid Arthritis is one of the most common autoimmune disorders. Drugs used are only to slow the progression and to enhance the quality of life. These therapies have several disadvantages as lack of selectivity and it can cause loss of patient compliance.

Aim: To identify the most common type of infection, rheumatoid arthritis patients in our region encountered, while using biologics and/or traditional DMARDs.

Method: Cross-sectional study was carried out in Rheumatology clinics at King Abdulaziz University Hospital, included Rheumatic patients who visit the clinics from 2013 to 2017. Data sheet was including demographic, using drug and American College of Rheumatology classification criteria for RA were added.

Result: Total of 164 patients were included in this study, 18 (11\%) males, and 146 (89\%) females. Trends among the target sample showed that $40(24.4 \%)$ had diabetes and 51(31.1\%) had hypertension. Patients who had been taking both DMARDs and Biologics, DRMADs only, biologics only were 49 (29.9\%), 74(45.1\%) and $15(9.1 \%)$ respectively. The total number of the infections was 180 , among them $62.78 \%$ were taking DMARDs only and the most common infection was lower urinary tract infections (UTI) 28(37.38\%).

Conclusion: UTI have the highest rate of infection in patients using traditional DMARDs and patient using both drug groups. And we recommend conducting a cohort study to identify these infections. It can help in assessing their outcomes.
\end{abstract}

Keywords: Rheumatoid Arthritis, biological drugs, DMARDs, infection

\section{INTRODUCTION}

Rheumatoid Arthritis (RA) is considered one of the most common human systemic autoimmune disorders. Therapies used now for RA patient are only to slow the progression of the disease and try to enhance the quality of life. However, these therapies have several disadvantages because of their lack of selectivity, and it can cause loss of patient compliance due to its frequent and long-term dosing ${ }^{(1)}$. The choice of treatment depends upon the disease state such as activity, prognosis regarding joint destruction, and complications (2). Disease Modifying AntiRheumatic Drugs (DMARDs) are used as the initial treatment they are effective by slowing down the progression and easing the symptoms. There are many different types of DMARDs including: methotrexate, leflunomide, hydroxychloroquine, and sulfasalazine. Methotrexate usually is the first medicine given, with another DMARD and a short course of corticosteroids to relieve any pain; or combined with biologics ${ }^{(3)}$. Biologics are made of proteins. They are potent and specific therapies; it works by antagonizing the mechanism of action of a particular chemical or cell involved in the occurrence of inflammation that results in joint swelling and other symptoms. While Traditional
DMARDs act non-specifically and have effects on different inflammation processes ${ }^{(4)}$.

There are ten biologics therapies approved for the treatment of RA, and they are usually used when treatment with DMARDs alone have failed. Half of these are anti-tumor necrosis factor (antiTNF) therapies: infliximab, adalimumab, golimumab, etanercept and certolizumab pegol. The first classes of biological therapies to be licensed for the treatment of RA were Anti-TNF therapies: Anakinra which works by blocking the interleukin-1 (IL-1) receptor. Abatacept which is a selective co-stimulator of T-cell activity, followed by rituximab which is an anti-B-cell therapy used in the management of non-Hodgkin's lymphoma. More recently, tocilizumab and Sarilumab were added which are considered as anti-IL-6 receptor antibodies, and, finally, tofacitinib which is a Janus kinase (JAK) inhibitor and this drug is the first orally administered biological agent ${ }^{(4)}$.

Due to the presence of different treatment options, clinicians need to compare between them and assess their efficacy and side effect. Infections are noted to be one of the most important risks in biological therapy, either serious or non-serious. Serious infection (SI) is defined by the FDA as the one that result in either death, hospitalization or the use of parenteral therapy. A meta-analysis state that 
the most common serious infections were UTI and respiratory infections ${ }^{(5)}$. Anti-TNF treatments are the most well studied, which can increase the risk of serious infections as an early side effect when it is compared to traditional DMARD - an outcome that was found in both experimental and observational studies ${ }^{(6-8)}$. Moreover, one of the most important risks that were increased by using anti-TNF therapies is tuberculosis, which is a very important disease in our country due to its high prevalence ${ }^{(4,9,10)}$. A single center prospective study on 89 patients with RA who is under the treatment of anti TNF blocking agents combined with corticosteroids, shows the rate of infections after six months: which was $10.3 \%$ in patients who used adalimumab, $12.8 \%$ with etanercept, and $19.04 \%$ with infliximab. And no hospitalization was observed in these three groups ${ }^{(11)}$.

On the opposite side, there are other studies that state; there is no increase in serious infection rate in the patient using anti-TNF therapy compared with traditional DMARDs, but there is an increase in severe skin and soft tissue infections ${ }^{(12)}$. A work of literature analyzed of 18 randomized clinical trials of a patient treated with anti-TNF therapy and found that with recommended doses the risk of serious infection is not increased. While in high doses there is a two-fold increase of serious infections risk ${ }^{(13)}$. A large, global clinical study on 4,789 patients with RA treated with tofacitinib which is a drug of the Janus Kinase is (JAK), had an increase in Herpes zoster cases compared with those using placebo. A percentage of $7 \%$ of $\mathrm{HZ}$ cases were serious, most of the patient either didn't discontinue the drug or discontinued temporarily $89 \%$ while $10 \%$ discontinued tofacitinib permanently due to the HZ event ${ }^{(14)}$.

There are no studies on biologics side effect on RA patients that was done in our area. That is why in this study we aim to identify the most common type of infection, rheumatoid arthritis patients in our region encountered, while using biologics and/or traditional DMARDs. The study was done in King Abdul-Aziz university hospital in Jeddah.

\section{PATIENTS AND METHODS}

Research design and setting: This crosssectional study was carried out at King Abdulaziz University Hospital (KAUH). KAUH is one of the biggest referral and teaching centers in the western region of Saudi Arabia with a capacity of 845 beds with the addition of 157 beds dedicated for the critical care units, general and specialized clinics that exceeds two hundred clinics. The study included Rheumatic patients (older than 18 years) in the Rheumatology clinics from 2013 to 2017. Consent was applied to every participant before the questionnaire was filled.

Participants: All adults' patient diagnosed with Rheumatoid Arthritis in King Abdulaziz University Hospital of both gender were included. Excluded the pregnant women due to their limited use of biological drugs and patient younger than 18 years.

Data collection method: This crosssectional study was carried out in Rheumatology clinics at King Abdulaziz University Hospital. The study included rheumatic patients (older than 18 years) who visit the clinics from 2013 to 2017.

Firstly, the demographic data: by asking about the typical questions like: (age, gender, nationality, patients phone number, educational level and smoking). Then, asking about any known medical disorders, the duration of rheumatoid arthritis and the using of glucocorticoid, biologics, traditional DMARDs and the name of drugs for each of them. All of these data were collected from the telephones. Also, by using the system of King abdulaziz university hospital we filled the anthropometrics measurements to assess nutritional status (Height which is done by the patient standing without shoes on a stadiometer, weight, and BMI was calculated as the patient's weight in $\mathrm{kg}$ divided by the square of the patient's height in $\mathrm{cm}$ ).

Data collection sheet was used, and 2 previous studies have been used as a source for writing this questionnaire ${ }^{(16,17)}$, the American College of Rheumatology (ACR) classification criteria for RA were added in the data collection sheet. Patient's records were also used for some lab results.

Data analysis: Data were coded, checked, and entered into SPSS version 22. For categorical variables, including gender, nationality, smoking, comorbidities, and disease duration categories were described using frequencies. While continuous variables including age, height and weight were described using mean and standard deviation. A chi-square was calculated to assess the associations between each categorical variable. For all statistical tests, $\mathrm{p}$ values less than 0.05 were considered significant. 


\section{RESULTS}

Out of total 173 patients participated in this study, nine patients were excluded due to missing data. So, complete data set was available for 164 patients during November of 2017. Among the sample, $18(11 \%)$ were males, and $146(89 \%)$ were females. In which 108 (65.9\%) were Saudis and 10 $(6.1 \%)$ were smokers. The mean age of the sample size was 49.6 ( \pm SD 12.97). The mean height was $158.8 \mathrm{~cm}$ ( \pm SD 15.97), and the mean weight was $73.85 \mathrm{~kg}( \pm$ SD21.9). Among the sample, 83 $(50.6 \%)$ had the RA induration less than five years, while $45(27.4 \%)$ had the disease in duration between 5 to 10 years, and $36(22 \%)$ had the disease in duration more than ten years.

Some of the trends among the target sample showed that 40 (24.4\%) had diabetes, 13 (7.9\%) had systemic lupus erythematosus, 51(31.1\%) had hypertension, 24 (14.6\%) had osteoporosis, 7(4.3\%) had Chronic Obstructive Pulmonary Disease, and 18 (11\%) had asthma. Regarding the drugs, 49 (29.9\%) of the patients were taking both DMARDs and Biologics, while $74(45.1 \%)$ were taking only DMARDs and $15(9.1 \%)$ of the patients were taking only Biologics. Among those on DMARDs, the most common drug used was Methotrexate 81 (49.3\%), while the most common drug used among those on Biologics was Adalimumab 22 (13.4\%). Among the total sample, 84(51.2\%) were taking Glucocorticoid.

We divided the sample into three categories according to the drug: DMARDs only, Biologics only, and combined (both DMARDs and Biologics). The total number of the infections was 180, among them $62.78 \%$ were taking DMARDs only, $37,22 \% \%$ were taking both DMARDs Biologics, and $17.22 \%$ were taking Biologics only.

Amongst patients on combined treatment (both DMARDs and Biologics), the most common infections were the urinary tract infection $17(34.69 \%)$, gastrointestinal infection 13(26.53\%), upper respiratory tract infection $13(26.53 \%)$, and skinlsubcutaneous tissue infection 10 (20.40\%). While patients who were taking Biologics only, the most common infection was upper respiratory tract infection $15(100 \%)$. Urinary tract infection 5 $(33.33 \%)$. Distribution of other infections in patients on Biologics only is shown in Table 1.

Among patients who were taking DMARDs only, the most common infections was lower urinary tract infection $28(37.38 \%$ ) the second is Upper respiratory tract infection 26 (35.13\%) and the Distribution of other infections in patients on DMARDs only is shown in Table 1 and distribution of the infections among the duration of RA is shown in Table 2.

\section{Appendix}

Table (1): Distribution of infections among type of treatment.

\begin{tabular}{|l|c|c|c|c|}
\hline & $\begin{array}{c}\text { BIOLOGICS } \\
(\mathbf{n = 1 5})\end{array}$ & $\begin{array}{c}\text { DRMADS } \\
(\mathbf{n = 7 4})\end{array}$ & $\begin{array}{c}\text { COMPANIED } \\
(\mathbf{n = 4 9})\end{array}$ & $\begin{array}{c}\text { Patients who } \\
\text { don't take } \\
\text { medications } \\
(\mathbf{n = 2 4})\end{array}$ \\
\hline $\begin{array}{l}\text { Any serious } \\
\text { infection (N } \\
(\%))\end{array}$ & 31 & 113 & 67 & 27 \\
\hline $\begin{array}{l}\text { Site of } \\
\text { infection (N } \\
(\%))\end{array}$ & & & & \\
\hline URTI & $15(100 \%)$ & $26(35.14 \%)$ & $13(19.40 \%)$ & 6 \\
\hline Bronchitis & $0(0 \%)$ & $4(5.41 \%)$ & $2(2.99 \%)$ & 1 \\
\hline Pneumonia & $1(6.67 \%)$ & $4(5.41 \%)$ & $3(4.48 \%)$ & 3 \\
\hline TB & $1(6.67 \%)$ & $4(5.41 \%)$ & $1(1.49 \%)$ & 1 \\
\hline HSV & $1(6.67 \%)$ & $7(9.46 \%)$ & $4(5.97 \%)$ & 1 \\
\hline HZV & $0(0 \%)$ & $2(2.70 \%)$ & $0(0 \%)$ & 1 \\
\hline Hepatitis & $0(0 \%)$ & $3(4.05 \%)$ & $0(0 \%)$ & 1 \\
\hline Candidiasis & $4(26.67 \%)$ & $15(20.27 \%)$ & $4(5.970 \%)$ & 2 \\
\hline UTI & $5(33.33 \%)$ & $28(37.84 \%)$ & $17(25.37 \%)$ & 7 \\
\hline GI infection & $2(13.33 \%)$ & $14(18.92 \%)$ & $13(19.40 \%)$ & 3 \\
\hline Bacteremia & $0(0 \%)$ & $1(1.35 \%)$ & $0(0 \%)$ & 0 \\
\hline $\begin{array}{l}\text { Skin } \\
\text { subcutaneous }\end{array}$ & $2(13.33 \%)$ & $5(6.77 \%)$ & $10(14.92 \%)$ & 1 \\
\hline
\end{tabular}

URTI: upper respiratory tract infection, TB: tuberculosis, HSV: Herpes simplex virus, HZV: Herpes zoster virus, UTI: urinary tract infection, GI: gastrointestinal

Table (2): URTI: Distribution of the infections among the duration of RA.

\begin{tabular}{|c|c|c|c|c|}
\hline & \multicolumn{3}{|c|}{ Duration of RA } & \multirow[b]{2}{*}{$\begin{array}{c}\mathbf{p} \\
\text { value }\end{array}$} \\
\hline & $\begin{array}{l}<5 \text { years } \\
N=(83)\end{array}$ & $\begin{array}{c}5-10 \text { years } \\
N=(45)\end{array}$ & $\begin{array}{l}>10 \text { years } \\
N=(36)\end{array}$ & \\
\hline URTI & $28(33.73 \%)$ & $8(17.78 \%)$ & $15(41.67 \%)$ & .428 \\
\hline Bronchitis & $4(4.82 \%)$ & $2(4.44 \%)$ & $1(2.78 \%)$ & .624 \\
\hline Pneumonia & $8(9.64 \%)$ & $4(8.89 \%)$ & $2(5.56 \%)$ & .086 \\
\hline TB & $5(6.02 \%)$ & $0(0 \%)$ & $3(8.33 \%)$ & .293 \\
\hline HSV & $6(7.24 \%)$ & $2(4.44 \%)$ & $5(13.89 \%)$ & .619 \\
\hline $\mathrm{HZV}$ & $1(1.20 \%)$ & $0(0 \%)$ & $2(5.56 \%)$ & .324 \\
\hline Hepatitis & $2(2.41 \%)$ & $1(2.22 \%)$ & $1(2.78 \%)$ & .987 \\
\hline Candida & $10(12.05 \%)$ & $5(11.11 \%)$ & $10(27.78 \%)$ & .301 \\
\hline UTI & $30(36.14 \%)$ & $10(22.22 \%)$ & $19(52.78 \%)$ & .272 \\
\hline GI infection & $17(20.48 \%)$ & $7(15.56 \%)$ & $8(22.22 \%)$ & .934 \\
\hline Bacteremia & $0(0 \%)$ & $0(0 \%)$ & $1(2.28 \%)$ & .183 \\
\hline $\begin{array}{l}\text { Skin } \backslash \\
\text { subcutaneous }\end{array}$ & $8(9.64 \%)$ & $6(13.33 \%)$ & $4(11.11 \%)$ & .462 \\
\hline
\end{tabular}

URTI: upper respiratory tract infection, TB: tuberculosis, HSV: Herpes simplex virus, HZV: Herpes zoster virus, UTI: urinary tract infection, GI: gastrointestinal

\section{DISCUSSION}

Patients with active rheumatoid arthritis have been associated with autoimmune disorders due to the effects of anti-TNF medications and its proinflammatory cytokines on macrophage. This pathogenesis will lead to developing multiple autoimmune disorders ${ }^{(15-17)}$. However, our data suggest that the presence of particular concern for 
the rheumatic patients who have the risk of developed diabetes mellitus type 2 . This increase in rheumatoid patients will lead to increase the risk of cardiovascular disorders. In comparison, the prevalence of CVD was higher in RA and diabetic by $12.4 \%$ in the DM2 group and $12.9 \%$ in those with RA, but the prevalence of CVD in nondiabetic group non- rheumatic patients was $5.0 \%$ ${ }^{(18)}$. Our results showed that the sample contains about $31.1 \%$ of rheumatic patients having hypertension followed with $24.4 \%$ having diabetes (19). Similar to our findings, another study showed hypertension and diabetes to be the most commonly occurring diseases in RA patients ${ }^{(20)}$.

The appropriate treatment like methotrexate with low dose corticosteroid has a significant role in increasing the risk of osteoporosis and reduce the bone mineral density for the advanced stages of RA; this hypothesis was applied in the S. Andrea University Hospital, Rome by Valentina Germano for 121 patients with RA between the 50s to 70s found prevalence rates of femoral neck osteoporosis and lumbar spine osteoporosis $(16.5 \%, \quad 23.1 \%$ respectively). The data reported in this study indicated that only age and body mass index (BMI) were significantly associated factors of osteoporosis in RA and the contribution of other factors of osteoporosis including disease activity, disease duration, and physical disability ${ }^{(22)}$. The results of our study showed a similar percentage of osteoporosis 24 (14.6\%).

In our study, DMARDs were more commonly used than Biologics, with percentages of $45.1 \%$ and $9.1 \%$ respectively. In this study DMARDs were associated with the highest rate of infections. Similar to our finding, a study showing similar results with higher rate of infection being associated with DMARDs ${ }^{(20)}$. On the other hand, Germano et al. showed that using DMARDs alone was associated with less infection rate in comparison to Biologics ${ }^{(23)}$. The latter, could be attributed to two reasons. Firstly, in our study we did not omit steroids effect; secondly, the number of patients using DMARDs is larger than those using Biologics.

Many of the cases, control studies were done at the same field and showed that the patients with active Rheumatoid arthritis have a higher risk of infections in comparison to the patients with active Osteoarthritis or either other Rheumatoid diseases ${ }^{(24)}$.
TNF has an essential role in controlling the risk of infection in the human body. Notably, TNF allows the macrophage for releasing in a maintenance way and forming the defense against the organism ${ }^{(23)}$. Tumor necrosis factor $\alpha$ (TNF $\alpha$ ) and interleukin 1 (IL 1) have an essential role in the management of rheumatoid arthritis (RA). They act by neutralizing the effects of proinflammatory cytokines. In particular, TNF inhibitors have a reverse effect for controlling the infection, and it plays a potential risk factor for developing the infection in animal models (24). These cytokine inhibitors like: (infliximab, etanercept, adalimumab, anakinra) are used to reduce the destruction of joints in patients with active disease. Nowadays, these drugs play a significant role in the treatment of RA, especially in patients who did not respond to disease modifying antirheumatic drugs (DMARDs).

Patients who are using biologic therapy have a higher prior risk of infection than those using other types of medications. However, our data suggest the presence of particular concern for upper respiratory tract infections. There was $100 \%$ increase in URTI among patients using biologics (TNF inhibitors). In addition, urinary tract infections were increased by $33.33 \%$. Data were available for 173 patients, only 15 patients were using Biologics agents. The results of the study were similar to the results of Joachim Listing study and Suzan M. Attar study ${ }^{(6,20)}$. The study estimated the incidence rate of serious and non-serious infections in patients with rheumatoid arthritis (RA) who were using biologics as a treatment.

Patients whom we failed to control their symptoms with only using one type of drugs, of either DMARDs or Biologics with the use steroids or not, we can try to control it by using both drug classes for a synergistic effect. For these patients, we wanted to see if the use of both drugs together had a more increase in the risk of infections. However, what was found is that the overall infection rate in patients who used combined drugs was not higher than patient using DMARDs. However, this lower prevalence can be contributed to the difference in a number of each sample.

Among the infections in a patient using combined drugs, UTI has the highest rate of infections $(34.69 \%)$, which is similar to a patient using DMARDs only. Followed by gastrointestinal infection and upper respiratory tract infection that are equal in prevalence $(26.53 \%)$. 
The causation of our study: Although our data collection was based on the telephone numbers, we took every single data and confirm it by hospital's system "phenix". Also there is some data we could not collect it from the patients themself like the lab results. so, we opened the hospital system to make sure about the results.Our sample size is huge in compared to other results. Regarding to the importance, our study will open the way to other researchers to generate new researches

Limitations of the study: This study faced some limitations due to its observational nature and telephone-based questionnaire. Firstly, we could not reach a significant number of patients, they either do not answer their phones, or they did not update their contact information in the hospital system. Secondly, there was recall bias which we could not overcome. Thirdly, many of the patients of low socioeconomic status, as a result, they did not know their medications names. A further potential limitation is the relatively small sample size as the study being applied in a single center.

\section{CONCLUSION}

In this study, we aimed to identify the most common type of infection in RA patient using biologics or traditional DMARDs. We concluded that urinary tract infections have the highest rate in patients using traditional DMARDs and patient using both drug groups. While patient using only biologics, URTI was the most common type. Overall the most common types of infection that were countered in RA patient using biologics or traditional DMARDs was UTI, URTI and GI infection.

For better assessment of infection risk in each type of drugs and the most common type of infection they encounter, a cohort study would be an advantageous method to identify these infections. It can also help in assessing their outcomes. And to see how this could affect the patients compliance to the medication.

\section{Acknowledgement:}

The authors would like to thank: Abeer Saleh Alghamdi,Bushra Suliman Almalki,Arwa Zuhair Fatani,Yara Mohammed Alamri,Haneen Abdullah Sahli,Buthainah Masoud Alghamdi, Anas Khalid Salman,Sarah Abdullah AlGhamdi,nourah ahmed aljehani,Douaa Mohammed Al-Bukhari, Maram Yahya Alqahtani,Bedoor khalid alshabibi, Albaraa mohammed altunisi,asalah sami felemban, Atheer Abdullah Al-Sulami, khulood ahmed althagafi, Rajwa Hassan Alobaidi. For their participation of data collector process.

\section{REFERENCES}

1. Gouveia VM, Lima SA, Nunes $C$, Reis $S$ (2015): Non-Biologic Nanodelivery Therapies for Rheumatoid Arthritis. J Biomed Nanotechnol., 11(10):1701-21.

2. Kumagai S (2015): Personalized Medicine in Rheumatoid Arthritis]. Rinsho Byori., 63(10):1175-81.

3. NHS(2016): Rheumatoid arthritis.Available from: https:// www. nhs. uk/ conditions/ rheumatoid-arthritis/treatment/.

4. Lahiri M, Dixon WG (2015): Risk of infection with biologic antirheumatic therapies in patients with rheumatoid arthritis. Best Pract Res Clin Rheumatol., 29(2):290-305.

5. Cipriani $P$, Berardicurti $O$, Masedu $F$, D'Onofrio F, Navarini L, Ruscitti $P$ et al. (2017): Biologic therapies and infections in the daily practice of three Italian rheumatologic units: a prospective, observational study. Clin Rheumatol.,36(2):251-60.

6. Listing J, Strangfeld A, Kary S, Rau R, von Hinueber U, Stoyanova-Scholz $M$ et al. (2005): Infections in patients with rheumatoid arthritis treated with biologic agents. Arthritis Rheum., 52(11):3403-12.

7. Bongartz T, Sutton AJ, Sweeting MJ, Buchan I, Matteson EL, Montori V (2006): Anti-TNF antibody therapy in rheumatoid arthritis and the risk of serious infections and malignancies: systematic review and metaanalysis of rare harmful effects in randomized controlled trials. JAMA.,295(19):2275-85.

8. Galloway JB, Hyrich KL, Mercer LK, Dixon WG, Fu B, Ustianowski AP(2011):Anti-TNF therapy is associated with an increased risk of serious infections in patients with rheumatoid arthritis especially in the first 6 months of treatment: updated results from the British Society for Rheumatology Biologics Register with special emphasis on risks in the elderly. Rheumatology (Oxford),50(1):124-31. 
9. Gómez-Reino JJ, Carmona L, Valverde VR, Mola EM, Montero MD (2003): Treatment of rheumatoid arthritis with tumor necrosis factor inhibitors may predispose to significant increase in tuberculosis risk: a multicenter active-surveillance report. Arthritis Rheum., 48(8):2122-7.

10. Listing J, Gerhold K, Zink A (2013): The risk of infections associated with rheumatoid arthritis, with its comorbidity and treatment. Rheumatology (Oxford), 52(1):53-61.

11. Benucci M, Saviola G, Baiardi P, Manfredi M, Sarzi Puttini P, Atzeni F (2012): Determinants of Risk Infection During Therapy with Anti TNF-Alpha Blocking Agents in Rheumatoid Arthritis. Open Rheumatol J.,6:33-7.

12. Dixon WG, Watson K, Lunt M, Hyrich KL, Silman AJ, Symmons DP et al. (2006): Rates of serious infection, including site-specific and bacterial intracellular infection, in rheumatoid arthritis patients receiving anti-tumor necrosis factor therapy: results from the British Society for Rheumatology Biologics Register. Arthritis Rheum., 54(8):2368-76.

13. Leombruno JP, Einarson TR, Keystone EC (2009): The safety of anti-tumour necrosis factor treatments in rheumatoid arthritis: meta and exposure-adjusted pooled analyses of serious adverse events. Ann Rheum Dis., 68(7):1136-45.

14. Winthrop KL, Yamanaka $H$, Valdez $H$, Mortensen E, Chew R, Krishnaswami S et al. (2014): Herpes zoster and tofacitinib therapy in patients with rheumatoid arthritis. Arthritis Rheumatol.,66(10):2675-84.

15. DM L, ME W(2001): Rheumatoid arthritis. Available from: http:// www. thelancet. com/ retrieve/pii/S0140673601060755.

16. Manicourt DH, Triki R, Fukuda K, Devogelaer JP, Nagant de Deuxchaisnes C, Thonar EJ (1993): Levels of circulating tumor necrosis factor alpha and interleukin-6 in patients with rheumatoid arthritis. Relationship to serum levels of hyaluronan and antigenic keratan sulfate. Arthritis Rheum., 36(4):490-9.

17. McInnes IB, Schett G (2011): The pathogenesis of rheumatoid arthritis. $\mathrm{N}$ Engl $\mathbf{J}$ Med. 365(23):2205-19.
18. Eid RE, Rao DA, Zhou J, Lo SF, Ranjbaran H, Gallo A et al. (2009): Interleukin-17 and interferon-gamma are produced concomitantly by human coronary artery-infiltrating $\mathrm{T}$ cells and act synergistically on vascular smooth muscle cells. Circulation, 119(10):1424-32.

19. Kitas GD, Erb $\mathbf{N}$ (2003): Tackling ischaemic heart disease in rheumatoid arthritis. Rheumatology (Oxford),42(5):607-13.

20. Attar A, Suzan M and Al Ghamdi A (2014): Rate of Infection in Rheumatoid Arthritis Patients. Saudi Journal of Internal Medicine, 41: 15-21.

21. Heidari B, Heidari P (2014): Bone mineral density loss in postmenopausal onset rheumatoid arthritis is not greater than premenopausal onset disease. Caspian J Intern Med., 5(4):213-8.

22. Germano V, Cattaruzza MS, Osborn J, Tarantino A, Di Rosa R, Salemi S et al. (2014): Infection risk in rheumatoid arthritis and spondyloarthropathy patients under treatment with DMARDs, corticosteroids and TNF- $\alpha$ antagonists. J Transl Med.,12:77.

23. Kolls JK, Lei D, Vazquez C, Odom G, Summer WR, Nelson S et al. (1997): Exacerbation of murine Pneumocystis carinii infection by adenoviral-mediated gene transfer of a TNF inhibitor. Am J Respir Cell Mol Biol., 16(2):112-8.

24. Arshad A, Rashid R (2008): The prevalence of bronchopulmonary infection among patients with rheumatoid arthritis versus non rheumatoid arthritis patients. Malays J Med Sci.,15(2): 24-28. 